\title{
Nitric oxide-mediated redox reactions in pathology, biochemistry and medicine
}

\author{
B Brüne $^{\star, 1}$ and $O$ Cantoni ${ }^{2}$ \\ ${ }^{1}$ Medical Department IV, Experimental Division, University of Erlangen-Nürnberg, Erlangen, Germany; ${ }^{2}$ Istituto di Farmacologia e Farmacognosia, University of Urbino, \\ Urbino, Italy \\ *Corresponding author: B Brüne, Faculty of Medicine, University of Erlangen-Nürnberg, Loschgestrasse 8, D-91054, Erlangen, Germany, \\ E-mail: Bernhard.Bruene@rzmail.uni-erlangen.de
}

Villa Vigoni Conference, Loveno di Menaggio, Italy, 12-15 April, 2000

The Italian-German Villa Vigoni conferences were established back in 1988 to discuss redox-reactions as mediators of cell signaling. Meetings are held consecutively every second to third year at the Villa Vigoni, Loveno di Menaggio, Italy, as a joint Italian-German event with roughly 15 participants from each side.

From the very beginning it has been the intention to exclude the traditional view of regarding reactive oxygen (ROS) and reactive nitrogen (RNS) species as merely unselective reactive byproducts of cellular metabolism that promote cell death. Initially, the role of oxidants, i.e. ROS in the regulation of physiological and pathological functions, particularly intracellular signal transduction, received considerable attention. With the notion that $\mathrm{NO}$ and its derived RNS appear as important inhibitors or activators of cell death, modulators of cell differentiation, and immune responses the complexity of NO-signaling, with an emphasis on ROS/RNS-interactions, became fundamental to our discussions.

The redox balance within a cell is determined by the level of prooxidant versus antioxidant molecules. The origin of ROS and RNS, which result in an oxidative challenge to the cell, is an important factor for the severity and duration of oxidative stress. $\mathrm{NO}$ is generated from the oxidation of $\mathrm{L}$ arginine to L-citrulline by a family of NO-synthase (NOS). The family consists of three isoenzymes; the constitutively expressed neuronal NOS (bNOS), the endothelial NOS (eNOS), and the cytokine-inducible NOS (iNOS), with the approximation that constitutive NOS-isoforms produce relative low and thus physiological amounts of $\mathrm{NO}$, whereas iNOS produces substantial, in part destructive amounts over an extended period of time. Sources for $\mathrm{O}_{2}-$ include, but are not limited to, mitochondrial respiration, arachidonate metabolizing enzymes, P450 mono-oxygenases, xanthine oxidases, and $\mathrm{NAD}(\mathrm{P}) \mathrm{H}$ oxidases. $\mathrm{NO}$ and $\mathrm{O}_{2}{ }^{-}$may be considered a nominal set of biologically relevant free radicals. Their interaction, reaction with oxidants and reductants yields any array of reactive species, only some of which are free radicals. Species being produced encompass among others compounds such as $\mathrm{NO}^{+}, \mathrm{NO}^{-}, \mathrm{ONOO}^{-}, \mathrm{N}_{2} \mathrm{O}_{3}$, or $\mathrm{H}_{2} \mathrm{O}_{2}$. RNS- and ROS-mediated reactions seem to utilize biochemical triggers such as oxidation (disulfide, mixed disulfide and/ or sulfenic acid formulation), S-nitrosation, or nitration of proteins to regulate an impressive and steadily growing number of normal and pathological functions in biological systems.

The role and mechanisms of $\mathrm{NO}$ and $\mathrm{O}_{2}{ }^{-}$in evoking cell death, apoptosis, or necrosis has governed growing attention over the past years. Expecially $\mathrm{NO}$ in its various redox forms can elicit or suppress the natural cell death program, apoptosis, in part as a result of its interaction with

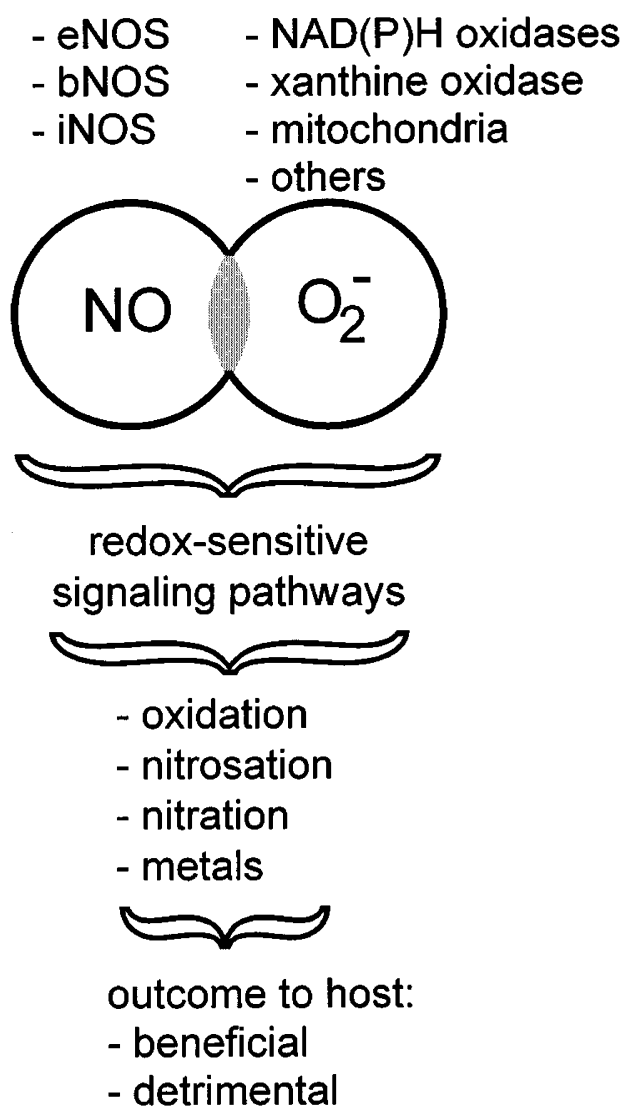

Figure 1 Sources and redox-signaling evoked by NO- and/or $\mathrm{O}_{2}{ }^{-}$. See text for details 
$\mathrm{O}_{2}{ }^{-}$. $\mathrm{NO}$, depending on its concentration, the biological redox milieu, and the involvement and/or induction of protective regimes can affect complex biological control mechanisms such as regulation of the vascular tone, cell demise under cellular conditions and in vivo, wound healing ischemia/reperfusion responses, cell respiration, activation/ deactivation of transcriptions factors, the phosphorylation/ dephosphorylation continuum, and stimulatory as well as inhibitory gene responses. In a few cases the source of $\mathrm{NO}$, the nature of target modification, and metabolic consequences are defined, whereas for other situations we are just at the beginning to get a detailed knowledge of redox reactions being involved. Examples discussed comprised:

\section{The vascular system}

The ERDF-like function of NO with concomitant relaxation of smooth muscle cells participates in regulating the vascular tone. As discussed by $\mathrm{V}$ Ullrich under conditions of increased $\mathrm{O}_{2}{ }^{-}$formation the generation of $\mathrm{ONOO}^{-}$will result in a rather selective nitration and inactivation of prostacyclin synthase at doses below $1 \mu \mathrm{M}$. The resultant accumulation of $\mathrm{PGH}_{2}$, as a consequence of inactivated prostacyclin synthase, further promotes vasocontraction. Due to the continued presence of $\mathrm{NO}$ it is hypothesized that the level of $\mathrm{O}_{2}{ }^{-}$determines the destructive nature in the vasculature. Generation of $\mathrm{O}_{2}{ }^{-}$is most likely achieved via activation of $\mathrm{NAD}(\mathrm{P}) \mathrm{H}$ oxidases. The $\mathrm{O}_{2}{ }^{-}$-generating enzyme is assembled by different components in endothelial versus smooth muscle cells, which may point to a selective activation/regulation process. One naturally occurring agonist that achieved $\mathrm{O}_{2}{ }^{-}$-generation is oxLDL, which deserves considerable attention for initiation and propagation of vascular injuries. $N A D(P) H$ oxidase activation may not only affect the equilibrium between NO and $\mathrm{O}_{2}{ }^{-}$and thus contractile responses, but may also activate the apoptotic program in endothelial cells by changing the ratio of pro- versus anti-apoptotic proteins, in part by affecting gene transcription.

\section{$\mathrm{ONOO}^{-}$signaling}

Besides its potential to nitrate e.g. prostacyclin synthase, somehow higher concentrations evoke complex signaling associated with receptor tyrosine nitration, activation of various mitogen activated protein kinases, or phospholipases with resultant arachidonate release. These effects may invoke inhibition of protein tyrosine phosphates via active site cysteine oxidation, which points to an indirect signaling pathway. So far the role of protein nitration as a prerequisite for a messenger function of $\mathrm{ONOO}^{-}$remains largely open. It appears essential to pinpoint targets for $\mathrm{ONOO}^{-}$that would allow to unravel its contribution as a signaling molecule and to define (pathological) situations that are causatively associated with these alterations.

\section{NO, a regulator of cell death and survival}

Based on the concept that NO may attenuate apoptosis by blocking caspases several in vitro and in vivo systems have been analyzed and discussed (E Clementi, A Wendel, G Melino). NO interferes with caspase activity in cell lysates of hepatocytes or Jurkat cells most likely by S-nitrosation with the notion that DTT fully reverses the NO-block. However, NO does not block apoptosis in hepatocytes when used in combination with TNF- $\alpha$ /actinomycine D. Moreover, NO exacerbates CD95/Fas-evoked hepatocyte apoptosis in vivo. In Jurkat cells the addition of $\mathrm{NO}$ together with Fas attenuated cell death. Under these conditions processing of caspases was attenuated and DTT was unable to regain enzymatic activity which explains decreased enzyme activity without blocking caspases by S-nitrosation. As a bottom line, one may conclude that inhibition of apoptosis by NO not necessarily reflects direct caspase modification. Rather, an interference at upstream signaling components needs consideration. Inhibition of DISC formation or antagonizing ceramide formation during TNF- $\alpha$ signaling may serve as an explanation. Along those lines, NO interferes with UVA/UVBmediated cell demise of endothelial cells. Induction of iNOS or application of $\mathrm{NO}$ donors prevented apoptosis as well as singulet oxygen-derived necrosis. Inhibition of cell death in this case arises from efficient antagonism of lipid peroxidation, an established feature of NO action. Inhibition of cell death by $\mathrm{NO}$ is contrasted by systems such as cardiomyocytes, macrophages, or neuronal cells where NO acts proapoptotic. Factors promoting cell death compromise the cGMPsignaling systems (in cardiomyocytes), and alterations in the expression of pro-versus anti-apoptotic proteins, among others p53, Bcl-2, or Bax. In general, the question was put forward whether blocking of apoptosis always appears rational (especially for slow degenerative diseases), since an interference with cell death execution pathways in non viable cells may favor inflammation instead of causing (tissue)-resolution (P Nicotera).

\section{Regulation of the hypoxic response (HIF-1 $\alpha$ accumulation)}

In various head and neck cancers there is an impressive correlation between iNOS-expression and tumor progression, with the finding that NOS-inhibitors provoke regression. NO promotes transcriptional control of VEGF expression (vascular endothelial growth factor) which appears as an important determinate of vascular angiogenesis. Additional observations pointed to the role of $\mathrm{NO}$ in modulating expression of the transcription factor HIF-1 $\alpha$, the classical hypoxia response factor. A putative oxygen sensor is supposed to produce ROS that promote destabilization of HIF-1 $\alpha$. Therefore, it sounds attractive to speculate that NO formation will redirect $\mathrm{O}_{2}{ }^{-}$signaling, thus allowing $\mathrm{HIF}-1 \alpha$ accumulation. NO-evoked HIF- $1 \alpha$ activation has been confirmed at the level of protein stabilization, by gel shift analysis, and reporter gene activation.

\section{Gene regulation}

Several studies established $\mathrm{NO}$ as well as $\mathrm{O}_{2}{ }^{-}$as efficient regulators of gene transcription and protein expression ( $\mathrm{J}$ Pfeilschifter, G Schettini). Among classical ROS-evoked responses one subsumes transcription factors such as AP-1 


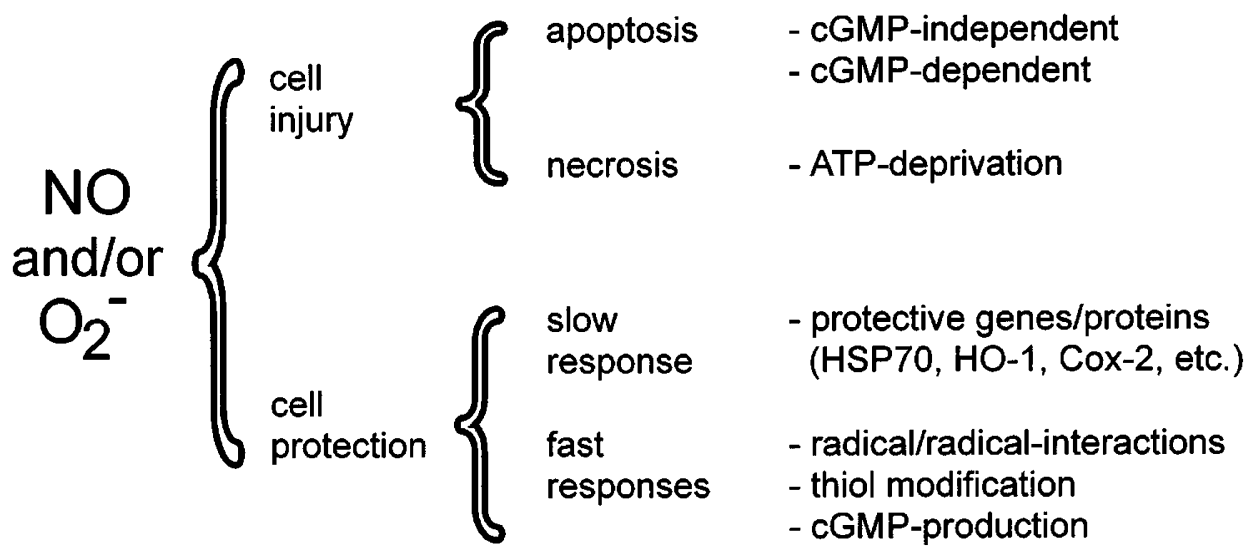

Figure 2 The role of $\mathrm{NO}$ and/or $\mathrm{O}_{2}{ }^{-}$in affecting cell demise. See text for details

and NF- $\kappa \mathrm{B}$. In fact RNS may use very similar pathways to achieve gene expression with the notion that $\mathrm{NO}$ and $\mathrm{O}_{2}{ }^{-}$ sometimes share an activating potency, whereas in other cases they behave contradictory. In many cases NO operates in a cGMP-independent manner, although examples of cGMP-evoked responses on expression of e.g. heme oxygenase-1 are known. As assumed for heme oxygenase1 , NO may protect cells by upregulating protective proteins or may use these pathways to provoke expression of antiinflammatory cytokines. A matter of controversy still is the contrasting response of $\mathrm{NO}$ on the transcription factor NF- $\kappa \mathrm{B}$. Activation and inhibition have been noticed. It will require further work to define conditions of $\mathrm{NO}$ in activating or blocking gene expression. The duration of the NO signal, the amount of NO being generated, the micro redox status, i.e. the absence or presence of $\mathrm{O}_{2}{ }^{-}$are considered as modulating factors. The challenging task in the future will be to identify a NO selective transcription factor (if one exists at all) or to elucidate the set of transcription factors being required for a NO-stimulatory response.

Redox controlled reactions participate in intracellular signaling, thus modulating diverse processes such as proliferation, differentiation, apoptosis, and necrosis. Oxidation may be shared by ROS as well as RNS, whereas nitration and nitrosation are thought to be RNS selective. It remains to be seen in how many ways proteins can integrate redox chemistry into functional responses and to delineate the selectivity of RNS versus ROS in achieving these alterations. The possibility that NO generation can contribute to the protection or vulnerability of cells requires our attention, especially when we consider the interaction of ROS and RNS under physiological and pathological conditions.

\section{Selected reading}

In the past several years state of the art editorials, letters, or reviews on multiple aspects of apoptosis have been published in Cell Death and Differentiation. For further reading on the role of NO in apoptosis, caspases, tumor suppressors, or Fas we refer to one of the following contributions:

Nicotera P, Bernassola F and Melino D (1999) Nitric oxide (NO), a signaling molecule with a killer soul. Cell Death Differ. 6: 931-933

Liu L and Stamler JS (1999) NO: an inhibitor of cell death. Cell Death Differ. 6: $937-$ 942

Lipton SA (1999) Neuronal protection and destruction by NO. Cell Death Differ. 6: 943-951

Li J and Billiar TR (1999) The anti-apoptotic actions of nitric oxide in hepatocytes. Cell Death Differ. 6: $952-955$

Dimmeler S and Zeiher AM (1999) Nitric oxide - an endothelial cell survival factor. Cell Death Differ. 6: $964-968$

Brüne B, von Knethen A and Sandau KB (1999) Nitric oxide (NO): an effector of apoptosis. Cell Death Differ. 6: 969-975

Thornberry NA (1999) Caspases: A decade of death research. Cell Death Differ. 6: $1023-1027$

Knight RA (1999) p53 and its younger siblings. Cell Death Differ. 6: 1143

Pinkoski MJ and Green DR (1999) Fas ligand, death gene. Cell Death Differ. 6: $1174-1181$

Samali A, Zhivotovsky B, Jones D, Nagata S and Orrenius S (1999) Apoptosis: Cell death defined by caspase activation. Cell Death Differ. 6: 495-496 